\title{
Expression of p16 and Survivin in gliomas and their correlation with cell proliferation
}

\author{
YANSHENG GAO ${ }^{1}$, LINGZHEN $\mathrm{LI}^{2}$ and LAIJUN SONG ${ }^{3}$ \\ Departments of ${ }^{1}$ Neurosurgery II and ${ }^{2}$ Neurology III, Zhumadian Central Hospital, Zhumadian, Henan 463000; \\ ${ }^{3}$ Department of Neurosurgery, The First Affiliated Hospital of Zhengzhou University, \\ Zhengzhou, Henan 450052, P.R. China
}

Received July 21, 2014; Accepted March 27, 2015

DOI: $10.3892 / \mathrm{ol} .2015 .3180$

\begin{abstract}
The survival rate of glioma patients is very low, and a lack of effective diagnostic techniques are available at present. The current study aimed to investigate the expression of p16 and Survivin and their association with proliferation and apoptosis in gliomas, as well as patient characteristics and prognosis. In total, 62 glioma specimens were surgically resected and pathologically confirmed at the Zhumadian Central Hospital (Zhumadian, China) between June 2008 and February 2014. Clinical data, including the gender and age of the patients, as well as the location, infiltration degree, size and pathological stage of the glioma, was collected. In order to evaluate the expression of p16 and Survivin in the gliomas, the Ki-67 labeling index was used to evaluate cell proliferation activity. The number of argyrophilic nucleolar organizer regions and the rate of cellular apoptosis was examined using the terminal deoxynucleotidyl transferase dUTP nick end labeling method. The results were analyzed using SPSS 14.0 statistical software. The positive rate of p16 gene expression in the gliomas was $46.77 \%$ (29 cases), and p16 gene expression was positively correlated with the differentiation status, tumor size and pre-operative symptoms. The positive rate of Survivin expression in the gliomas was $69.88 \%$ (58 cases), and Survivin expression was positively correlated with tumor size, differentiation status and clinical stage. The proliferation activity of the gliomas was enhanced with increasing p16 and Survivin expression, while apoptosis was inhibited. In conclusion, the overexpression of p16 and Survivin was closely associated with uncontrolled cell proliferation and the inhibition of apoptosis in gliomas. The combined analysis of the expression of p16 and Survivin in gliomas may provide guidance with respect to the clinical diagnosis, evaluation, treatment and prognosis of patients with glioma.
\end{abstract}

Correspondence to: Dr Yansheng Gao, Department of Neurosurgery II, Zhumadian Central Hospital, 747 Zhonghua Road, Zhumadian, Henan 463000, P.R. China

E-mail: yanshenggao@yeah.net

Key words: glioma, p16, Survivin, proliferation activity

\section{Introduction}

Gliomas of the brain originate from brain glial cells and may be benign or malignant. Often, the tumors are located in important regions of the brain and no clear boundaries exist between the glioma cells and the normal brain tissue. Therefore, the complete removal of the glioma by surgical resection can be challenging. In certain circumstances, surgery is not a suitable approach. However, radiotherapy and chemotherapy are not effective strategies for the treatment of gliomas. Due to the presence of the blood brain barrier, the majority of chemical drugs and Chinese anti-tumor medicines are also ineffective. Therefore, gliomas demonstrate one of the worst prognoses of all tumors $(1,2)$. When gliomas initially arise, there are usually no evident symptoms. As the tumor develops, symptoms, including an increase of the intracranial pressure accompanied by headache, emesis, hypopsia, diplopia, epilepsy and psychiatric symptoms, are observed. In addition, local symptoms are generated as a result of oppression, infiltration or damage to the brain tissue, depending on the location of the tumor (3).

With the development of clinical technologies, the early diagnosis and cure rates of gliomas have improved significantly (3). However, the survival rate of the majority of glioma patients remains low. Further investigation is required in order to improve the efficacy of glioma treatments, particularly those aimed at malignant gliomas, and also to identify novel therapeutic approaches. The mechanisms underlying the pathogenesis of gliomas also require further investigation. However, in the majority of cases, the early diagnosis and classification of gliomas remains challenging. The diagnosis of gliomas relies upon a combination of clinical, radiological and pathological methods $(4,5)$. The present study aimed to evaluate the expression of p16 and Survivin in gliomas, and investigate their correlation with cell proliferation. Immunohistochemistry was also used to investigate the role of p16 and Survivin in the development of gliomas. The results of the present study may provide guidance with respect to the clinical diagnosis, assessment, treatment and prognosis of gliomas.

\section{Subjects and methods}

Subjects. In total, 62 glioma specimens were obtained from patients diagnosed following surgical resection at the 
Zhumadian Central Hospital (Zhumadian, China) between June 2008 and February 2014 for the present study. Of the 62 patients recruited, 37 were male and 25 were female, with a mean age of $50.17 \pm 8.13$ years. The present study was conducted in accordance with the declaration of Helsinki and with approval from the Ethics Committee of Zhumadian Central Hospital. Written informed consent was obtained from all participants.

According to the World Health Organization (WHO) 1999 classification (6), gliomas may be divided into various types, namely astrocytomas, oligodendrogliomas, ependymomas, mixed gliomas, choroid plexus papillomas, neural epithelial tumors of uncertainty origin, mixed tumors of neuron and neuronal glial, pineal parenchymal tumors, embryonic tumors and neuroblastoma tumors. According to the WHO 2000 classification (6), gliomas are divided into low-grade gliomas (LGG) and high-grade gliomas (HGG). LGG include pathological stage I-II gliomas, whereas HGG include pathological stage III-IV gliomas (7). The glioma types in the present study included 14 cases of astrocytoma, 8 cases of oligodendroglioma, 8 cases of ependymoma, 10 cases of mixed glioma, 6 cases of choroids plexus papilloma, 6 cases of mixed neuronal and neuronal glial tumor, 5 cases of pineal parenchymal tumors, 3 cases of embryonic tumor and 2 cases of neuroblastoma tumor. In total, 23 of these cases were classified as stage I-II and 39 as stage III-IV.

Experimental design. In total, 62 patients with brain gliomas participated in the present study. The clinical and follow-up data (collected three months after discharge from the hospital) of these patients were analyzed retrospectively. On the basis of previous studies, the indexes of interest, which included age, gender, tumor size, pre-operative symptoms, differentiation, metastasis and malignant degree, were selected for statistical analysis. The resected glioma specimens obtained from the 62 patients were formalin-fixed, paraffin-embedded and sliced into $4-\mu \mathrm{m}$ serial sections. Following dewaxing and dehydration, the sections were flushed with phosphate-buffered saline. Next, a polymer enhancer (Boster Biological Technology, Ltd., Wuhan, China), rabbit polyclonal antibody against human Ki-67 (catalog no. 26921; Daan Gene Co., Ltd, Guangzhou, China; dilution, 1:200; incubation, $37^{\circ} \mathrm{C}, 10 \mathrm{~min}$ ) and a monoclonal mouse anti-human cyclin antibody (catalog no. 26908; Daan Gene Co., Ltd.; dilution, 1:100) were added to the slides, and the sections were incubated for $1 \mathrm{~h}$ at room temperature. The sections were then washed three times with phosphate-buffered saline, followed by incubation with horseradish peroxidase-conjugated monoclonal goat anti-rabbit or anti-mouse (catalog no. 9103; Cell Signaling Technology, Inc., Beverly, MA, USA; dilution, 1:2,000) secondary antibodies for $30 \mathrm{~min}$ at $37^{\circ} \mathrm{C}$. Finally, 3,3'-diaminobenzidine chromogenic liquid (Daan Gene Co., Ltd.) was added. Following staining with hematoxylin, the slices were sealed with neutral resin. Subsequent to specimen processing, the expression of p16 and Survivin in the glioma tissues was observed under a microscope (BX50; Olympus Corporation, Tokyo, Japan).

Diagnostic criterion. According to the criteria of the 1996 National Symposium of Immunohistochemistry Technology (8), if the number of immunoreactive positive cells is $>25 \%$ in ten continuous high-power magnification ( $x 40$ ) fields, the specimen is considered to be positive for the expression of a particular protein (1) For p16, the presence of a yellow or brown nucleus under the microscope indicated positive expression (2) For Survivin, the presence of brown particles in the cytoplasm indicated a positive cell.

One-step silver staining. Cellular proliferation was examined using the one-step silver staining method. The detailed process, including the staining and counting of the number of argyrophilic nucleolar organizer regions have been previously reported (4).

Terminal deoxynucleotidyl transferase dUTP nick end labeling. Cellular apoptosis was detected using the In situ Cell Death Detection kit (Boehringer Mannheim, Mannheim, Germany). The apoptotic nuclei exhibited a blue color. The number of apoptotic cells was calculated using an optical microscope (BX51; Olympus Corporation; magnification, $\mathrm{x} 400$ ) with a grid micrometer (Olympus Corporation; number per $0.25 \mathrm{~mm}^{2}$ ).

Clinical factor analysis. On the basis of previous studies $(3,4)$, clinical factors of the glioma patients, including gender, age, invasion depth, distant metastasis, differential degree, tumor size and clinical stage, were evaluated. In addition, the correlations between clinical factors and p16 or Survivin expression were also analyzed in the present study.

Statistical analysis. Statistical analyses were performed using SPSS 14.0 (SPSS Inc., Chicago, IL, USA). All data are expressed as the mean \pm standard deviation. The associated risk factors for glioma were analyzed by stepwise regression. The comparison of the positive expression rates of $\mathrm{p} 16$ and Survivin protein, in addition to cellular proliferation and apoptosis, was conducted using the $\chi^{2}$ test. A value of $\mathrm{P}<0.05$ was considered to indicate a statistically significant difference.

\section{Results}

p16 expression is correlated with tumor size, differential degree and pre-operative conditions. Of the 62 glioma patients, the total positive rate of p16 protein expression was $46.77 \%$ ( 29 cases). Through analyzing the correlation between p16 protein expression and the clinical factors of the glioma patients, it was revealed that p16 had no significant association with gender, age, invasion depth, distant metastasis or clinical stage $(P>0.05)$. However, a significant correlation was identified between p16 expression and the differential degree, tumor size and pre-operative conditions $(\mathrm{P}<0.05)$. The positive expression rate of $\mathrm{p} 16$ was significantly lower $(26.31 \%)$ in patients with highly-differentiated gliomas than in those with lowly-differentiated gliomas (55.81\%). The positive expression rate of p16 in patients with tumors measuring $>4 \mathrm{~cm}$ in size (66.67\%) was markedly higher than those with tumors measuring $\leq 4 \mathrm{~cm}$ in size (36.59\%). The positive expression rate of $\mathrm{p} 16$ in patients with pre-operative symptoms $(53.06 \%)$ was markedly higher than those without pre-operative symptoms (23.08\%). A significant statistical difference was identified between patients with and without pre-operative symptoms ( $\mathrm{P}<0.05$; Fig. 1A; Table I).

Survivin expression is correlated with tumor size, differential degree and clinical stage. Of the 62 glioma patients 
Table I. Correlation between p16 and Survivin expression and the clinical factors of glioma patients.

\begin{tabular}{|c|c|c|c|c|c|c|c|c|c|}
\hline \multirow[b]{2}{*}{ Clinical factor } & \multirow[b]{2}{*}{ Patients, $\mathrm{n}$} & \multicolumn{3}{|c|}{ p16 protein expression } & \multirow[b]{2}{*}{ P-value } & \multicolumn{3}{|c|}{ Survivin protein expression } & \multirow[b]{2}{*}{ P-value } \\
\hline & & $(-), \mathrm{n}$ & $(+), \mathrm{n}$ & Positive rate, $\%$ & & $(-), \mathrm{n}$ & $(+), \mathrm{n}$ & Positive rate, $\%$ & \\
\hline Total & 62 & 33 & 29 & & & 27 & 35 & & \\
\hline Age, years & & & & & 0.847 & & & & 0.608 \\
\hline$<60$ & 33 & 17 & 16 & 48.48 & & 13 & 20 & 60.60 & \\
\hline$\geq 60$ & 29 & 16 & 13 & 44.83 & & 14 & 15 & 51.72 & \\
\hline Gender & & & & & 0.872 & & & & 0.753 \\
\hline Male & 37 & 20 & 17 & 45.94 & & 17 & 20 & 54.05 & \\
\hline Female & 25 & 13 & 12 & 48.00 & & 10 & 15 & 60.00 & \\
\hline Tumor size, $\mathrm{cm}$ & & & & & $0.039^{\mathrm{a}}$ & & & & $0.023^{\mathrm{b}}$ \\
\hline$\leq 4$ & 41 & 26 & 15 & 36.59 & & 23 & 18 & 43.90 & \\
\hline$>4$ & 21 & 7 & 14 & 66.67 & & 4 & 7 & 80.95 & \\
\hline $\begin{array}{l}\text { Pre-operative } \\
\text { symptoms }\end{array}$ & & & & & $0.007^{\mathrm{a}}$ & & & & 0.243 \\
\hline No & 13 & 10 & 3 & 23.08 & & 7 & 6 & 46.25 & \\
\hline Yes & 49 & 23 & 26 & 53.06 & & 20 & 29 & 59.18 & \\
\hline Distant metastasis & & & & & 0.841 & & & & 0.779 \\
\hline No & 10 & 5 & 5 & 50.00 & & 4 & 6 & 60.00 & \\
\hline Yes & 52 & 28 & 24 & 46.15 & & 23 & 29 & 55.77 & \\
\hline Clinical stage & & & & & 0.213 & & & & $0.004^{\mathrm{b}}$ \\
\hline I-II & 23 & 15 & 8 & 34.78 & & 16 & 7 & 30.43 & \\
\hline III-IV & 39 & 18 & 21 & 53.85 & & 11 & 28 & 71.79 & \\
\hline Differentiation status & & & & & $0.009^{\mathrm{a}}$ & & & & $0.029^{b}$ \\
\hline High & 19 & 14 & 5 & 26.31 & & 12 & 7 & 36.84 & \\
\hline Low & 43 & 19 & 24 & 55.81 & & 15 & 28 & 65.11 & \\
\hline
\end{tabular}

${ }^{\mathrm{a}} \mathrm{P}<0.05$, the expression level of $\mathrm{p} 16$ was significantly different between two groups; ${ }^{\mathrm{b}} \mathrm{P}<0.05$, the expression level of Survivin was significantly different between two groups.

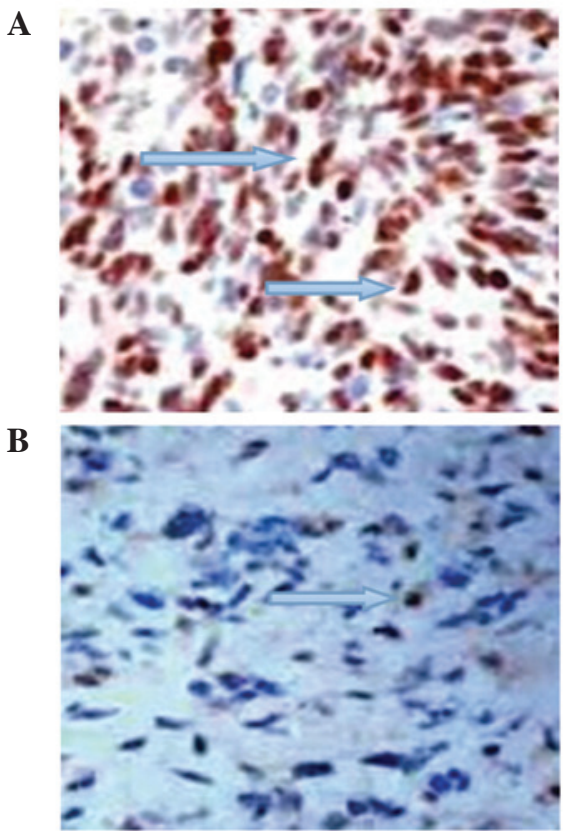

Figure 1. p16 and Survivin are expressed in glioma tissues. Positive expression of (A) p16 and (B) Survivin in glioma tissue (magnification, x200). Arrows indicate positive cells. analyzed, 35 demonstrated positive expression of Survivin protein $(56.45 \%)$. Through analyzing the correlation between Survivin protein expression and the clinical factors of the patients with glioma, it was revealed that Survivin was not significantly associated with gender, age, distant metastasis or pre-operative status ( $\mathrm{P}>0.05)$. However, Survivin expression was significantly correlated with tumor size, differential degree and clinical stage $(\mathrm{P}<0.05)$. In patients with a high differentiation status, a tumor size of $\leq 4 \mathrm{~cm}$ or a stage I-II tumor, the positive expression rate of Survivin was 36.84, 43.90 and $30.43 \%$, respectively. However, in patients with a low differentiation status, a tumor size of $>4 \mathrm{~cm}$ or a stage III-IV tumor, the positive expression of Survivin was $65.11,80.95$ and $71.79 \%$, respectively. The differences between these two groups were significant $(\mathrm{P}<0.05$; Fig. 1B; Table I).

p16 protein expression is correlated with enhanced cellular proliferation and decreased apoptosis. In patients exhibiting positive expression of p16 protein, cellular proliferation was significantly higher than that in patients with a negative expression of p16 protein $(\mathrm{P}<0.05)$. By contrast, the number of apoptotic cells was significantly lower in patients with a positive 
Table II. Correlation between p16 protein and cellular proliferation and apoptosis.

\begin{tabular}{lcccc}
$\begin{array}{l}\text { p16 } \\
\text { expression }\end{array}$ & $\mathrm{n}$ & Ki-67 LI & AgNOR & Apoptotic cells, n \\
\hline$(+)$ & 29 & $11.37 \pm 1.05$ & $4.14 \pm 0.58$ & $26.59 \pm 7.18$ \\
$(-)$ & 33 & $6.09 \pm 2.57$ & $2.73 \pm 1.55$ & $57.71 \pm 27.09$ \\
F-value & & $5.77^{\mathrm{a}}$ & $4.13^{\mathrm{a}}$ & $6.48^{\mathrm{a}}$ \\
\hline
\end{tabular}

${ }^{\mathrm{a}} \mathrm{P}<0.05$. LI, labeling index; AgNOR, argyrophilic nucleolar organizer regions. Values are presented as the mean \pm standard deviation.

p16 protein expression. The differences between these two groups were statistically significant $(\mathrm{P}<0.05$; Table II).

Survivin protein expression is correlated with enhanced cellular proliferation and suppressed apoptosis. In patients exhibiting positive expression of Survivin protein, cellular proliferation levels were significantly higher than those in patients with a negative expression of p16 protein $(\mathrm{P}<0.05)$. By contrast, the number of apoptotic cells was significantly lower in patients with a positive expression of Survivin protein $(\mathrm{P}<0.05$; Table III).

Survivin and p16 expression are correlated. Through analyzing the correlation between Survivin and p16 in patients with gliomas, it was revealed that the expression levels of Survivin and p16 exhibited a significant association between the two factors $(\mathrm{R}=0.758 ; \mathrm{P}<0.05)$, which indicated that the selection of these factors in the present study was appropriate.

\section{Discussion}

Glioma accounts for $45 \%$ of all intracranial tumors, and is ranked the second most common malignancy amongst children. During the last 30 years, the incidence of primary malignant brain tumors has increased each year, particularly in the elderly population, with an annual growth rate of $\sim 1.2 \%$ worldwide. According to the literature, the annual incidence of glioma in China is 3-6/100,000. The annual number of mortalities occurring as a result of gliomas is 30,000 . According to a report published by the WHO, malignant glioma is the second leading cause of mortality in cancer patients $<35$ years old. Furthermore, it is estimated that $400,000-600,000$ people succumb to malignant glioma annually $(9,10)$.

The diagnosis of glioma should be based upon the biological characteristics and location of the tumor, the age and gender of the patient and the clinical course. Following the obtainment of a medical history and a review of the symptoms, electrophysiology, ultrasound, radionuclide, radiology, nuclear magnetic resonance and laboratory examinations should be applied, which are able to provide a positioning accuracy of almost $100 \%$ and a diagnosis rate of $>90 \%$. The efficacy of glioma-targeted therapies is determined by the stage of the disease and the treatment approach used. Early detection is important in order to improve the efficacy of treatment and the survival rate of patients $(4,5)$.

In China, $<10 \%$ of patients with glioma are diagnosed at an early stage. The main reason for this is that patients often
Table III. Correlation between Survivin protein and cellular proliferation and apoptosis.

\begin{tabular}{lcccc}
$\begin{array}{l}\text { Survivin } \\
\text { expression }\end{array}$ & $n$ & Ki-67 LI & AgNOR & Apoptotic cells, n \\
\hline$(+)$ & 35 & $8.79 \pm 3.37$ & $3.46 \pm 1.02$ & $33.83 \pm 17.39$ \\
$(-)$ & 27 & $4.15 \pm 2.27^{\mathrm{a}}$ & $2.25 \pm 0.70^{\mathrm{a}}$ & $58.91 \pm 25.22^{\mathrm{a}}$ \\
F-value & & $6.04^{\mathrm{b}}$ & $4.67^{\mathrm{b}}$ & $4.38^{\mathrm{b}}$ \\
\hline
\end{tabular}

${ }^{\mathrm{a}} \mathrm{P}<0.05 ;{ }^{\mathrm{b}} \mathrm{P}<0.01$. LI, labeling index; AgNOR, argyrophilic nucleolar organizer regions. Values are presented as the mean \pm standard deviation.

only seek medical advice following the onset of discomfort. However, the symptoms of glioma usually only begin to appear at the middle or late stage of the disease course (11).

With the development of molecular biology techniques facilitating the study of genes and proteins, significant results have been obtained concerning the mechanisms that underlie the pathogenesis of gliomas. The diagnosis and treatment of gliomas has thus entered a new era. Clinical studies have revealed that gliomas with identical classifications may react differently to the same treatment (12). Furthermore, prognoses, as well as the infiltration, recurrence and metastasis of the tumor, differ (13). Studies have confirmed that differences amongst gliomas with identical histological subtypes are due to alterations in gene expression. The application of molecular biological techniques and immunohistochemistry is therefore important in order to detect these altered gene or protein levels (14). In clinical practice, physicians should use these test results, combined with clinical data, surgical cases and the routine pathological diagnosis of patients, to develop optimized individualized comprehensive treatment plans. With this approach, the treatment of gliomas may be standardized. Previous studies have confirmed that p16 and Survivin genes have significant roles in the formation and development of certain malignant tumors $(15,16)$. Therefore, the p16 and Survivin genes were selected for investigation in the present study.

The p16 tumor suppressor gene, also known as the multiple tumor suppressor 1 gene, was discovered by Kamb (17) in Cold Spring Harbor Laboratory (Cold Spring Harbor, NY, USA) in 1994. The deletion of homozygous p16 was detected in 50\% of human tumor cell lines. p16 is a basic gene involved in the regulation of the cell cycle, where it inhibits cellular proliferation and division. Failure of the p16 gene may lead to the malignant proliferation of cells and the formation of a tumor. Missense and frameshift mutations have been identified in a number of malignant tumors, including brain tumors, as well as lung, skin, breast, bone and bladder cancer, which indicates that a deletion or mutation of the p16 gene may contribute to the growth and development of tumors (18). At present, p16 is considered to be a more significant anticancer gene than $\mathrm{p} 53$. Therefore, the identification of changes in the $\mathrm{p} 16$ gene has important clinical implications when determining the susceptibility and prognosis of a tumor. Furthermore, detecting the expression of p16 protein in glioma tissues is 
critical for classifying the malignant and metastatic degree of the glioma. The results of the present study revealed that the expression of the p16 protein was not significantly associated with patient gender or age, the depth of invasion, metastasis or the clinical stage; however, it was significantly correlated with the degree of differentiation, tumor size and pre-operative symptoms. In patients with highly-differentiated gliomas, the positive expression rate of p16 (26.31\%) was significantly lower than that of patients with a lowly-differentiated glioma $(55.81 \%)$. The positive expression rate of p16 in patients with a tumor size of $>4 \mathrm{~cm}(66.67 \%)$ or with pre-operative symptoms $(53.06 \%)$ was significantly higher than that in patients with a tumor size of $\leq 4 \mathrm{~cm}(36.59 \%)$ or without pre-operative symptoms $(23.08 \%)(\mathrm{P}<0.05)$. The results indicated that the positive expression of p16 was closely associated with tumor differentiation status and size, as well as pre-operative symptoms. The lower the glioma differentiation and the larger the tumor size, the higher the positive expression rate of p16 detected. This suggested that p16 may be valuable in guiding clinical treatments and predicting the prognosis of gliomas. Hassounah et al (19) found that there were $>50 \%$ p16 homozygous deletions in cystic tumors, bone tumors and lymphoma, as well as lung, breast, renal, ovarian, skin and bladder cancer, and that nonsense, missense and frameshift mutations were present in melanomas. These results suggested that the p16 gene participates in the formation of various tumor tissues. The presence of mutations and deletions in the p16 gene may be an important index to predict the nature and prognosis of tumors.

The Survivin protein is a novel inhibitor of the apoptosis protein family. It is believed to be the most effective apoptosis inhibitor. Survivin has a number of complex functions, including the inhibition of cellular apoptosis and the promotion of cell transformation. In addition, Survivin is involved in cell mitosis, the formation of blood vessels and the resistance of tumors to drugs. At present, Survivin has gained marked interest in the field of tumor research. The protein is tumor specific, which means that it is only expressed in tumor and embryonic tissues, and is closely associated with tumor differentiation, proliferation, invasion and metastasis $(20,21)$. The expression of the Survivin gene has been identified to be upregulated in nasal type natural killer (NK)/T cell lymphoma cells and has been found to be significantly correlated with the expression of p53 and B cell lymphoma 2 proteins. Survivin is believed to be an important marker for classifying the prognosis of gliomas (22). The results of the present study revealed that the positive expression rate of Survivin protein was not significantly correlated with gender, age, distant metastasis or pre-operative conditions ( $\mathrm{P}>0.05)$, but was significantly associated with the tumor size, differential degree and clinical stage $(\mathrm{P}<0.05)$. In patients who presented with a glioma that was highly-differentiated, $\leq 4 \mathrm{~cm}$ in size or classified as stage I-II, the positive expression rate of Survivin was $36.84,43.90$ and $30.43 \%$, respectively. However, in patients with low differentiation, a tumor size of $>4 \mathrm{~cm}$ or a glioma that was classified as stage III-IV, the positive expression of Survivin was $65.11,80.95$ and $71.79 \%$, respectively. The differences between the two groups were significant $(\mathrm{P}<0.05)$. These results suggested that Survivin may be used as a marker to indicate the differentiation degree and clinical stage of gliomas. Detecting the expression of
Survivin may be valuable for predicting the invasion and metastasis of a glioma, and the development and prognosis of patients following surgery. In addition, the expression level of the Survivin gene has previously been demonstrated to be significantly upregulated in nasal type NK/T cell lymphoma cells, which suggests that Survivin may be used as a reference index in order to predict the prognosis of glioma patients.

Under physiological conditions, cellular proliferation and apoptosis are in a dynamic equilibrium. In the present study, it was revealed that the protein expression levels of p16 and Survivin were significantly upregulated, cell proliferation was increased and cell apoptosis was significantly decreased. p16 proteins, which were demonstrated to be positive by immunohistochemistry, lose their ability to inhibit cell division and induce cell apoptosis as a result of Survivin upregulation $(23,24)$. In addition, the high expression levels of Survivin protein inhibits cell apoptosis and promotes tumor growth $(23,24)$. Thus, we hypothesize that in the present study, although the expression of p16 was identified, normal p16 function was inhibited as a result of Survivin upregulation, leading to the increased cell proliferation observed. A previous study identified that high expression levels of Survivin were closely associated with mutated p16 and the formation and development of gliomas (25). Mutation of the p16 gene enhances the induction effect of protein kinase $C$ on the expression of Survivin (26). The present study also demonstrated that the expression of Survivin was positively correlated with p16, which suggested that the expression of Survivin may be regulated by p16. It has been established that the overexpression of Survivin and p16 is associated with the malignant transformation of a number of tumors $(27,28)$.

The present study investigated the expression of p16 and Survivin in gliomas, and their correlation with cell proliferation. The results indicated that the proliferative activity of the gliomas was enhanced with increasing p16 and Survivin expression, while apoptosis was inhibited. The findings of the present study may provide guidance for the clinical diagnosis, condition assessment, treatment and prognosis of gliomas.

\section{References}

1. Roci E, Cakani B, Brace G, Bushati T, Rroji A, Petrela M and Kaloshi G: Platinum-based chemotherapy in recurrent high-grade glioma patients: Retrospective study. Med Arch 68: 140-143, 2014.

2. Feng B, Hu P, Lu SJ, Chen JB and Ge RL: Increased argonaute 2 expression in gliomas and its association with tumor progression and poor prognosis. Asian Pac J Cancer Prev 15: 4079-4083, 2014.

3. Sai K, Yang QY, Shen D and Chen ZP: Chemotherapy for gliomas in mainland China: An overview. Oncol Lett 5: 1448-1452, 2013.

4. Watts C, Price SJ and Santarius T: Current concepts in the surgical management of glioma patients. Clin Oncol (R Coll Radiol) 26: 385-394, 2014.

5. Ahmed R, Oborski MJ, Hwang M, Lieberman FS and Mountz JM: Malignant gliomas: Current perspectives in diagnosis, treatment and early response assessment using advanced quantitative imaging methods. Cancer Manag Res 6: 149-170, 2014.

6. Kleihues P, Louis DN, Scheithauer BW, Rorke LB Reifenberger G, Burger PC and Cavenee WK: The WHO classification of tumors of the nervous system. J Neuropathol Exp Neurol 61: 215-225, 2002.

7. Adida C, Crotty PL, McGrath J, Berrebi D, Diebold J and Altieri DC: Developmentally regulated expression of the novel cancer anti-apoptosis gene survivin in human and mouse differentiation. Am J Pathol 152: 43-49, 1998. 
8. Editorial Committee of Chinese Journal of Pathology: The meeting summary of the national symposium on immunohistochemical technique and diagnosis standard. Zhonghua Bing Li Xue Za Zhi 25: 326-328, 1996 (In Chinese).

9. Shankar SL, Mani S, O'Guin KN, Kandimalla ER, Agrawal S and Shafit-Zagardo B: Survivin inhibition induces human neural tumor cell death through caspase-independent and -dependent pathways. J Neurochem 79: 426-436, 2001.

10. Parenti A, Leo G, Porzionato A, Zaninotto G, Rosato A and Ninfo V: Expression of survivin, p53 and caspase 3 in Barrett's esophagus carcinogenesis. Hum Pathol 37: 16-22, 2006.

11. Pan Y, Hu WH, Xie D, et al: Nuclear and cytoplasmic expressions of survivin in glioma and their prognostic value. Zhonghua $\mathrm{Yi}$ Xue Za Zhi 87: 325-329, 2007 (In Chinese).

12. Ene CI and Holland EC: Personalized medicine for gliomas. Surg Neurol Int 6 (Suppl 1): S89-S95, 2015.

13. Wilden JA, Voorhies J, Mosier KM, O'Neill DP and Cohen-Gadol AA: Strategies to maximize resection of complex, or high surgical risk, low-grade gliomas. Neurosurg Focus 34: E5, 2013 .

14. Honda R, Körner R and Nigg EA: Exploring the functional interactions between Aurora B, INCENP and survivin in mitosis. Mol Biol Cell 14: 3325-3341, 2003.

15. Al-Khalaf HH, Lach B, Allam A, et al: Expression of survivin and $\mathrm{p} 16(\mathrm{INK} 4 \mathrm{a}) / \mathrm{Cdk} 6 / \mathrm{pRB}$ proteins and induction of apoptosis in response to radiation and cisplatin in meningioma cells. Brain Res 1188: 25-34, 2008.

16. Chung $\mathrm{CH}$, Zhang Q, Kong CS, et al: p16 protein expression and human papillomavirus status as prognostic biomarkers of nonoropharyngeal head and neck squamous cell carcinoma. J Clin Oncol 32: 3930-3938, 2014.

17. Kamb A: Role of a cell cycle regulator in hereditary and sporadic cancer. Cold Spring Harb Symp Quant Biol 59: 39-47, 1994.

18. Söling A, Plugge EM, Schmitz M, et al: Autoantibodies to the inhibitor of apoptosis protein survivin in patients with brain tumors. Int J Oncol 30: 123-128, 2007.
19. Hassounah M, Lach B, Allam A, et al: Benign tumors from the human nervous system express high levels of survivin and are resistant to spontaneous and radiation-induced apoptosis. J Neurooncol 72: 203-208, 2005.

20. Bongiovanni L, Di Diodoro F, Della Salda L and Brachelente C: On the role of survivin as a stem cell biomarker of canine hair follicle and related tumours. Vet Dermatol 25: 138-141, 2014.

21. Khan S, Bennit HF, Turay D, Perez M, Mirshahidi S, Yuan Y and Wall NR: Early diagnostic value of survivin and its alternative splice variants in breast cancer. BMC Cancer 14 176,2014

22. Kogiku M, Ohsawa I, Matsumoto K, Sugisaki Y, Takahashi H, Teramoto A and Ohta S: Prognosis of glioma patients by combined immunostaining for survivin, Ki-67 and epidermal growth factor receptor. J Clin Neurosci 15: 1198-1203, 2008.

23. Saito T, Arifin MT, Hama S, et al: Survivin subcellular localization in high-grade astrocytomas: Simultaneous expression in both nucleus and cytoplasm is negative prognostic marker. J Neurooncol 82: 193-198, 2007.

24. Nandi S, Ulasov IV, Tyler MA, et al: Low-dose radiation enhances survivin-mediated virotherapy against malignant glioma stem cells. Cancer Res 68: 5778-5784, 2008.

25. Pennati M, Folini M and Zaffaroni N: Targeting survivin in cancer therapy. Expert Opin Ther Targets 12: 463-476, 2008.

26. Chen LH, Li YW, Gao LY and Liu JF: An evaluation for the function and significance concerned to alternations of p16 3D structure with gene mutation in esophageal squamous cell carcinoma. Chin J Med Genet 23: 208-212, 2006.

27. Selemetjev S, Dencic TI, Marecko I, Jankovic J, Paunovic I, Savin S and Cvejic D: Evaluation of survivin expression and its prognostic value in papillary thyroid carcinoma. Pathol Res Pract 210: 30-34, 2014.

28. Piaton E, Carré C, Advenier AS, et al: p16 INK4a overexpression and p16/Ki-67 dual labeling versus conventional urinary cytology in the evaluation of urothelial carcinoma. Cancer Cytopathol 122: 211-220, 2014. 